\title{
Subcision Using a Spinal Needle Cannula and a Thread for Prominent Nasolabial Fold Correction
}

\author{
Sang-Yeul Lee ${ }^{1}$, Kun-Yong Sung ${ }^{2}$ \\ ${ }^{1}$ Department of Plastic and Reconstructive Surgery, Kangwon National University College of Medicine, Chuncheon; ${ }^{2}$ Department of Plastic \\ and Reconstructive Surgery, Kangwon National University Hospital, Kangwon National University College of Medicine, Chuncheon, Korea
}

Deepening of the nasolabial crease is an esthetically unpleasing aging phenomenon occurring in the midface. Various treatment modalities have been introduced to improve the appearance of prominent nasolabial folds, all of which have pros and cons. Currently, a minimally invasive technique using synthetic dermal fillers is most commonly used. A simple and easy subcision procedure using a wire scalpel has also been used and reported to be effective for prominent nasolabial fold correction, with minimal complications. As an alternative to the wire scalpel, we used a 20-gauge metal type spinal needle cannula (Hakko Co.) and 4-0 Vicryl suture (Ethicon Inc.) for subcision of nasolabial folds. This technique is less expensive than the use of a wire scalpel and easily available when needed. Therefore, on the basis of favorable results, our modified subcision technique may be considered effective for prominent nasolabial fold correction.

Keywords Nasolabial fold / Sutures / Dissection

\author{
Correspondence: Kun-Yong Sung \\ Department of Plastic and \\ Reconstructive Surgery, Kangwon \\ National University Hospital, \\ Kangwon National University College \\ of Medicine, 156 Baekryeong-ro, \\ Chuncheon 200-722, Korea \\ Tel: +82-33-258-9494 \\ Fax: +82-33-258-9437 \\ E-mail: hanyangps@naver.com
}

This study was supported by a 2012 Kangwon National University Hospital Grant.

No potential conflict of interest relevant to this article was reported.

Received: 21 Jan 2013 • Revised: 24 Feb 2013 • Accepted: 26 Feb 2013

pISSN: 2234-6163 • elSSN: 2234-6171 • http://dx.doi.org/10.5999/aps.2013.40.3.256• Arch Plast Surg 2013;40:256-258

\section{INTRODUCTION}

The nasolabial fold first becomes obvious around the age of 25 years and becomes more apparent with aging, although there is great individual variation [1]. To improve the appearance of prominent nasolabial folds, various treatment modalities such as synthetic dermal fillers, fat grafts, allogenic or xenogenic materials, and alloplastic implants have been used, with variable results [2]. The subcision technique has also been used for prominent nasolabial fold correction. Orentreich and Orentreich [3] introduced subcision using tribeveled hypodermic needles and reported it to be effective in correcting various types of skin depressions. Thereafter, several authors [4-7] reported that subcision with a wire scalpel was effective for depressed scars, wrinkles, and folds. As an alternative to the wire scalpel method for nasolabial fold correction, we describe herein a modified subcision technique using a 20-gauge metal type spinal needle cannula (Hakko Co., Chikuma, Japan) and 4-0 Vicryl suture (Ethicon Inc., Somerville, NJ, USA).

\section{Surgical technique}

The area to be subcised (about 7 to $8 \mathrm{~mm}$ wide) was outlined preoperatively while the patient was in a sitting position. A $1 \%$ lidocaine solution containing 1:100,000 epinephrine was infiltrated subcutaneously under the operative fields. The 20-gauge spinal needle cannula was inserted upward through point $A$ and passed through the subdermal plane along the medial line of the designated area (Fig. 1A); the cannula was then made to exit 


\section{Fig. 1. Surgical technique}

(A) Insertion of the 4-0 Vicryl suture through the 20-gauge spinal needle cannula. (B) Reinsertion of the thread through the cannula tip. (C) "Back and forth" movement with countertraction by the assistant.
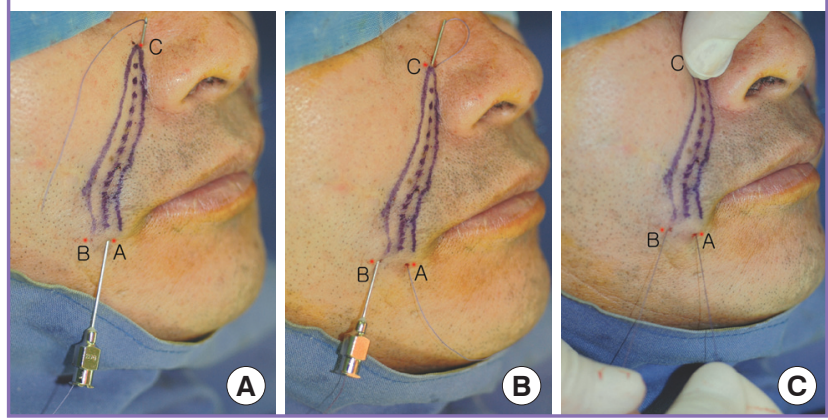

\section{Fig. 2. Case 1}

The 58-year-old female patient who underwent a single thread subcision for bilateral folds and simultaneous nasal tip plasty. (A) Preoperative photograph. (B) Two-year postoperative photograph.
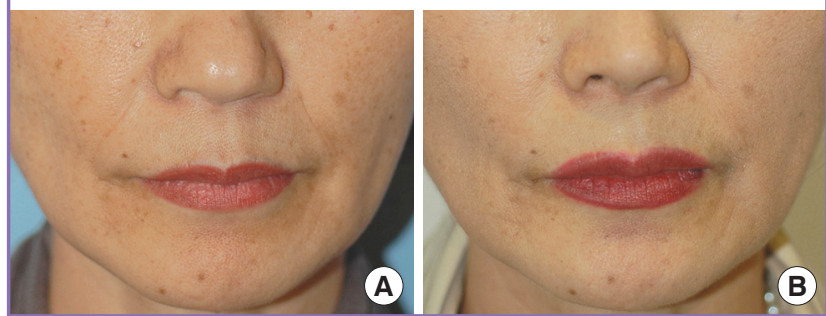

at endpoint $\mathrm{C}$, the proximal nasolabial fold. After inserting the thread into the cannula head, the thread was pushed up gently until its end passed through the cannula tip. The spinal needle cannula was removed and then introduced through point $\mathrm{B}$. The cannula was passed through the same plane along the lateral line of the designated area until the cannula tip was made to exit at point $\mathrm{C}$. The thread, which had already been drawn out of point $\mathrm{C}$, was reinserted into the cannular tip until the thread came out through the cannula head (Fig. 1B). After removing the spinal needle cannula, the outlined area was then undermined with a "back and forth" motion with countertraction by the assistant (Fig. 1C). While performing the "back and forth" motion, resistance could often be felt at the modiolus. After completing the procedure, ice compression was applied for 20 minutes.

\section{CASE}

\section{Case 1}

A 58-year-old female patient underwent a single procedure of bilateral thread subcision for prominent nasolabial fold correction and simultaneous nasal tip plasty. She encountered no complications and was satisfied with the postoperative results (Fig. 2).

\section{Fig. 3. Case 2}

The 46-year-old female patient who underwent a single thread subcision for bilateral fold. (A) Preoperative photograph. (B) Fivemonth postoperative photograph.
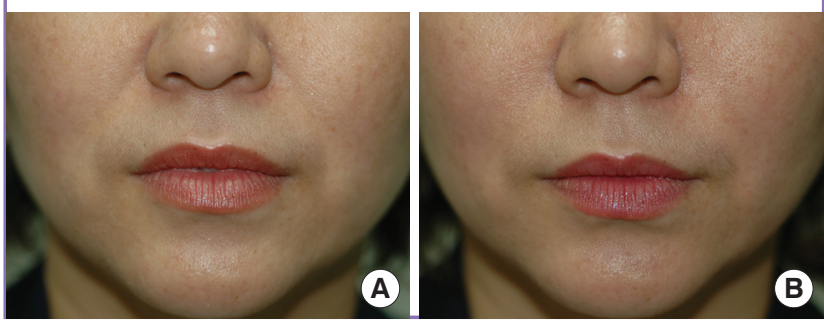

\section{Case 2}

A 46-year-old female patient underwent a single procedure of bilateral thread subcision to improve the appearance of her nasolabial folds. Favorable results were obtained without complications (Fig. 3).

\section{DISCUSSION}

Prominent nasolabial folds can be esthetically improved using various treatment modalities. To obtain optimal results, the treatment plan should be individualized according to the patient's age, aesthetic needs, anticipated downtime, and even economic status [2]. Subcision, which is incisionless subcuticular undermining, was first introduced by Orentreich and Orentreich [3]. The authors reported that subcision with tribeveled hypodermic needles was effective in correcting various types of skin depressions. They postulated that a depression could be lifted by the releasing action of the procedure and the formation of fibrotic tissue in the normal course of wound healing. The individual propensity for fibroplasia in the subcised area depends upon skin tension, which may cause internal hypertrophic scarring [4]. The wire scalpel instrument consisting of a braided wire attached to a straight needle that was introduced by Sulamanidze et al. [7] has been used for subcision in the clinical field. The authors concluded that subcutaneous dissection using a wire scalpel is a simple, safe, and effective method for improving the appearance of scars or age-related contour defects. Several other authors [4-6] have reported that subcision with a wire scalpel is effective for depressed scars, wrinkles, or folds. Furthermore, they reported that better results were obtained by simultaneous filling with fat $[5,6]$ or other autogenous tissues [4] after subcision. To improve the appearance of nasolabial folds, we used a modified subcision technique using a 20 -gauge spinal needle cannula and a 4-0 Vicryl suture, which are less expensive and easily accessible when a wire scalpel is not available. The use of a cannula with a larger than 20-gauge bore might be more conve- 
nient but has a higher possibility of leaving a needle mark. The 4-0 Vicryl suture is durable enough to overcome the resistance at the modiolus during subcision. Mutifilament sutures such as Vicryl seem more proper for tissue sawing than monofilament sutures.

This procedure, which we call thread subcision, can be applied in patients who want prominent nasolabial fold correction alone; it can be performed in bilateral nasolabial folds as well as in a unilateral fold for asymmetric fold correction. It can also be performed as an ancillary procedure to a face-lift operation for better correction of nasolabial folds. We have used a wire scalpel before for the same purpose. The reason that we use thread instead of a wire scalpel is because thread is less expensive and more easily available in South Korea than wire scalpels. Based on our clinical experience, thread subcision was demonstrated to be as effective as wire scalpel subcision in improving the appearance of nasolabial folds. To obtain optimal results from subcision, it is essential to induce the formation of adequate amounts of scar tissue in the subcised area. The amount of fibroplasia after subcision varies with skin tension and the number of subcision procedures [3]. When optimal results cannot be obtained with a single procedure, the procedure can be repeated at regular intervals of 2 to 3 months for more favorable results. In addition, simultaneous intradermal subcision using a hypodermic needle might be helpful to improve the appearance of nasolabial folds in patients with fine wrinkles. The width of the subcised area might also influence fibroplasia. Hence, we suggest that approximately 7 to $8 \mathrm{~mm}$ is an adequate width for the subcision area. More extensive undermining might induce hematoma and even internal hypertrophic scarring, whereas less extensive undermining causes undercorrection. There were only minor complications such swelling, bruising, and minor irregularities. However, they resolved spontaneously with conservative management. Based on the favorable results of our study, our modified subcision technique may be considered effective for nasolabial fold correction. However, further study of the various factors influencing fibroplasia is needed to obtain better and more consistent results from subcision.

\section{REFERENCES}

1. Guyuron B, Michelow B. The nasolabial fold: a challenge, a solution. Plast Reconstr Surg 1994;93:522-9.

2. Lee SY. Prominent nasolabial fold: an overview of treatments. Arch Aesthetic Plast Surg 2011;17:143-52.

3. Orentreich DS, Orentreich N. Subcutaneous incisionless (subcision) surgery for the correction of depressed scars and wrinkles. Dermatol Surg 1995;21:543-9.

4. Sasaki GH. Comparison of results of wire subcision performed alone, with fills, and/or with adjacent surgical procedures. Aesthet Surg J 2008;28:619-26.

5. Kotlus BS, Dryden RM. Modification of the nasolabial crease with wire scalpel and autologous fat transfer. Am J Cosmet Surg 2006;23:75-8.

6. Graivier M. Wire subcision for complete release of depressions, subdermal attachments, and scars. Aesthet Surg J 2006;26:387-94.

7. Sulamanidze MA, Salti G, Mascetti M, et al. Wire scalpel for surgical correction of soft tissue contour defects by subcutaneous dissection. Dermatol Surg 2000;26:146-50. 